\title{
An example of a bireflectional spin group
}

\section{Journal Article}

Author(s):

Villa, Oliver

Publication date:

2003-07

Permanent link:

https://doi.org/10.3929/ethz-b-000053964

Rights / license:

In Copyright - Non-Commercial Use Permitted

Originally published in:

Archiv der Mathematik 81(1), https://doi.org/10.1007/s00013-003-0817-x 
Arch. Math. 81 (2003) 1-4

0003-889X/03/010001-04

DOI 10.1007/s00013-003-0817-x

(C) Birkhäuser Verlag, Basel, 2003

Archiv der Mathematik

\title{
An example of a bireflectional spin group
}

\author{
By
}

OLIVER VILLA

\begin{abstract}
Let $\mathfrak{n}$ be the anisotropic norm of a Cayley algebra $\mathfrak{C}$ over a field $F$ of characteristic different from 2 where -1 is a square. Let $\operatorname{Spin}(\mathfrak{C}, \mathfrak{n})$ be the spin group of the quadratic form $\mathfrak{n}$. We prove that every element in $\operatorname{Spin}(\mathfrak{C}, \mathfrak{n})$ is a product of two involutory elements, i.e. $\operatorname{Spin}(\mathfrak{C}, \mathfrak{n})$ is bireflectional.
\end{abstract}

Introduction. A group $G$ is called bireflectional if every element is a product of two involutory elements of $G$. Here we call $g \in G$ an involutory element if $g^{2}=1$. Bachmann suggested to investigate the bireflectionality in the classical groups: the bireflectionality in the orthogonal, symplectic and unitary groups has been studied in many cases (see [2]). On the other hand, to my knowledge, there are no results on the bireflectionality in the spin group of a quadratic form (however, in [1], it was proved that any element in the spin group of a quadratic form, defined over a finite field of odd characteristic, can be written as product of three involutory elements). In this note we prove that the spin group of the anisotropic norm of a Cayley algebra over a field $F$ of characteristic different from 2 is bireflectional if -1 is a square in $F$. The proof goes as follows: for the spin group of the norm of a Cayley algebra there is a very particular description; we use this description and the fact that in our case the special orthogonal group is bireflectional.

1. The description of the spin group. The material considered in this section may be found in [4]. For the convenience of the reader we give a brief survey of the notations of [4] that we adopt. Let $F$ be a field of characteristic different from 2 and $V$ a finite dimensional $F$-vector space. Let $q: V \longrightarrow F$ be a quadratic form. We define the symmetric bilinear form $b_{q}: V \times V \longrightarrow F$ by $b_{q}(x, y):=q(x+y)-q(x)-q(y)$ for $x, y \in V$, and we assume that $b_{q}$ is nondegenerate, i.e. $b_{q}(x, y)=0 \forall y \in V$ implies $x=0$.

Let $A=\operatorname{End}_{F}(V)$ be the split central simple algebra. The adjoint anti-involution $\sigma_{q}$ : $A \longrightarrow A$ is defined by the equation

$$
b_{q}(x, f(y))=b_{q}\left(\sigma_{q}(f)(x), y\right) \text { for } x, y \in V, f \in A .
$$


The Clifford algebra of a quadratic form $q: V \longrightarrow F$ is denoted by $C(q)$, the even Clifford algebra by $C_{0}(q)$ (see $\left.[4,8 . \mathrm{A}]\right)$.

We consider a Cayley algebra $\mathfrak{C}$ over $F$ with conjugation $\pi: x \mapsto \bar{x}$ and norm $\mathfrak{n}: x \mapsto x \bar{x}$. For the basic facts on Cayley algebras we refer to [4, Chapter VIII]. The norm $\mathfrak{n}$ is a quadratic form defined on $\mathfrak{C}$ (as in [4], we also denote by $\mathfrak{C}$ the vector space structure of the Cayley algebra $\mathfrak{C}$ ). We recall that the spin group of $\mathfrak{n}: \mathfrak{C} \longrightarrow F$ is defined by

$$
\operatorname{Spin}(\mathfrak{C}, \mathfrak{n}):=\left\{c \in C_{0}(\mathfrak{n}) \mid c \cdot \tau(c)=1 \quad \text { and } \quad c \cdot x \cdot c^{-1} \in \mathfrak{C} \quad \forall x \in \mathfrak{C}\right\},
$$

where $\tau$ is the involution induced by the identity on $\mathfrak{C}$ and the symbol $\cdot$ denotes the multiplication in the Clifford algebra. To describe the group $\operatorname{Spin}(\mathfrak{C}, \mathfrak{n})$, we define the new multiplication $x \star y=\bar{x} \bar{y}$. Let $r_{x}(y):=y \star x$ and $l_{x}(y):=x \star y$. Then the map $\mathfrak{C} \longrightarrow \operatorname{End}_{F}(\mathfrak{C} \oplus \mathfrak{C})$ given by

$$
x \mapsto\left(\begin{array}{cc}
0 & l_{x} \\
r_{x} & 0
\end{array}\right)
$$

induces isomorphisms of algebras with involution (see [4, 35.1]):

$$
\alpha:(C(\mathfrak{n}), \tau) \longrightarrow\left(\operatorname{End}_{F}(\mathfrak{C} \oplus \mathfrak{C}), \sigma_{\mathfrak{n} \perp \mathfrak{n}}\right)
$$

and

$$
\alpha_{0}:\left(C_{0}(\mathfrak{n}), \tau\right) \longrightarrow\left(\operatorname{End}_{F}(\mathfrak{C}), \sigma_{\mathfrak{n}}\right) \times\left(\operatorname{End}_{F}(\mathfrak{C}), \sigma_{\mathfrak{n}}\right)
$$

Let $\mathrm{O}^{+}(\mathfrak{n})$ denote the special orthogonal group (i.e. the group of orthogonal transformations with determinant equal to 1$)$. A triple $\left(t_{1}, t_{2}, t_{3}\right) \in \mathrm{O}^{+}(\mathfrak{n})^{3}$ will be called related if $t_{1}(x \star y)=t_{2}(x) \star t_{3}(y)$ for all $x, y \in \mathfrak{C}$. We define the group $\operatorname{RT}(\mathfrak{C}, \mathfrak{n}):=\left\{\left(t_{1}, t_{2}, t_{3}\right) \in\right.$ $\mathrm{O}^{+}(\mathfrak{n})^{3} \mid\left(t_{1}, t_{2}, t_{3}\right)$ is related $\}$.

For every element $c \in \operatorname{Spin}(\mathfrak{C}, \mathfrak{n})$, we let $\alpha_{0}(c)=\left(\begin{array}{cc}t_{3} & 0 \\ 0 & t_{2}\end{array}\right)$.

Let $\chi_{c}(x)=c \cdot x \cdot c^{-1}$ be the vector representation.

Proposition 1.1. The map

$$
\Psi: \operatorname{Spin}(\mathfrak{C}, \mathfrak{n}) \longrightarrow \operatorname{RT}(\mathfrak{C}, \mathfrak{n})
$$

defined by $\Psi(c):=\left(\chi_{c}, t_{2}, t_{3}\right)$ is an isomorphism of groups.

Proof. See $[4,35.7]$.

2. Bireflectionality. Let $F$ be a field of characteristic not 2 and assume that -1 is a square in $F$, i.e. there is an element $s \in F$ with $s^{2}=-1$. We consider a Cayley algebra $\mathfrak{C}$ over $F$ with anisotropic norm $\mathfrak{n}$.

Lemma 2.1. If $\left(t_{1}, t_{2}, t_{3}\right)$ is a related triple and $t_{1}^{2}=1$, then $t_{2}^{2}=t_{3}^{2}=1$. 
Pro of. If $\left(t_{1}, t_{2}, t_{3}\right)$ is a related triple, then $\left(t_{1}^{2}, t_{2}^{2}, t_{3}^{2}\right)$ is a related triple, too. Moreover, the pair $\left(t_{2}, t_{3}\right)$ is determined by $t_{1}$, up to a factor $\left(\mu, \mu^{-1}\right), \mu \in F^{\times}$. The fact that $t_{2} \in \mathrm{O}^{+}(\mathfrak{n})$ implies $\mu^{2}= \pm 1$. Since $t_{1}^{2}=1$ and since the triple $(1,1,1)$ is related, we conclude that $t_{2}^{2}=t_{3}^{2}= \pm 1$. Suppose that $t_{2}^{2}=-1$. Pick an anisotropic vector $x$. The vectors $x$ and $t_{2}(x)$ are orthogonal:

$$
b_{\mathfrak{n}}\left(x, t_{2}(x)\right)=b_{\mathfrak{n}}\left(t_{2}(x), t_{2}^{2}(x)\right)=-b_{\mathfrak{n}}\left(x, t_{2}(x)\right) .
$$

Since -1 is a square, the plane generated by $x$ and $t_{2}(x)$ is hyperbolic, a contradiction to the anisotropy of $\mathfrak{n}$. Hence $t_{2}^{2}=t_{3}^{2}=1$.

Theorem 2.2. The group $\operatorname{Spin}(\mathfrak{C}, \mathfrak{n})$ is bireflectional.

Proof. Let $c \in \operatorname{Spin}(\mathfrak{C}, \mathfrak{n})$ with $\Psi(c)=\left(t_{1}, t_{2}, t_{3}\right)$.

Since $\operatorname{dim} \mathfrak{C}=8 \equiv 0 \bmod 4$, then, by $[3,3.2]$, there are two involutory elements $\sigma_{1}$ and $\tau_{1}$ in $\mathrm{O}^{+}(\mathfrak{n})$ with

$$
t_{1}=\sigma_{1} \tau_{1}
$$

By triality (see $[4,35.4])$, there are maps $\sigma_{2}, \sigma_{3}$ in $\mathrm{O}^{+}(\mathfrak{n})$ such that the triple $\left(\sigma_{1}, \sigma_{2}, \sigma_{3}\right)$ is related. But then the triple $\left(\sigma_{1} t_{1}, \sigma_{2} t_{2}, \sigma_{3} t_{3}\right)=\left(\tau_{1}, \sigma_{2} t_{2}, \sigma_{3} t_{3}\right)$ is related, too. By 2.1 ,

$$
\left(\sigma_{i}\right)^{2}=1, \quad\left(\sigma_{i} t_{i}\right)^{2}=1, \quad i=2,3 .
$$

We conclude that

$$
\Psi(c)=\left(\sigma_{1}, \sigma_{2}, \sigma_{3}\right)\left(\tau_{1}, \sigma_{2} t_{2}, \sigma_{3} t_{3}\right),
$$

as desired.

Ex a m ple 2.3. Let $\mathbb{C}$ be the field of complex numbers. The number -1 is a square in the field $\mathbb{C}\left(x_{1}, x_{2}, x_{3}\right)$. The quadratic form

$$
\mathfrak{n}=\left\langle 1, x_{1}\right\rangle \otimes\left\langle 1, x_{2}\right\rangle \otimes\left\langle 1, x_{3}\right\rangle
$$

over $\mathbb{C}\left(x_{1}, x_{2}, x_{3}\right)$ is anisotropic and is the norm of a Cayley algebra $\mathfrak{C}$. Hence $\operatorname{Spin}(\mathfrak{C}, \mathfrak{n})$ is bireflectional.

Acknowledgement. I wish to thank Prof. Erich W. Ellers for his comments and suggestions.

\section{References}

[1] P. C. Austin and E. W. Ellers, Products of Involutions in the Finite Chevalley Groups of type $F_{4}(K)$. Comm. Algebra 30(8), 4019-4029 (2002).

[2] E. W. ELLERS, Bireflectionality in Classical Groups. Canad. J. Math. 29(6), 1157-1162 (1977). 
[3] F. KNUPPEL and K. NiELSEN, Products of Involutions in $\mathrm{O}^{+}(V)$. Linear Algebra Appl. 94, 217-222 (1987).

[4] M. -A. Knus, A. Merkurjev, M. Rost and J. -P. Tignol, The Book of Involutions. Amer. Math. Soc. Colloq. Publ. 44 (1998).

Received: 28 February 2002

Oliver Villa

ETH Zentrum Zürich

CH-8092 Zürich

Switzerland 